\title{
Dual Stent-Assisted Coil Embolization for Fusiform Aneurysm Arising From Persistent Trigeminal Artery
}

\author{
Gordhan Ajeet, MD', Dickinson John, DO²
}

Aneurysms of the persistent trigeminal artery (PTA) trunk are exceptionally rare and have a high risk for rupture. Dual stent placement through each internal carotid and basilar artery for endovascular coil embolization of a fusiform aneurysm arising from the PTA has not been described in the literature. A 44year-old female with a history of chronic headache was identified to have a fusiform aneurysm arising from medially coursing adult type, Saltzman type 3 PTA trunk. Sacrifice of the PTA trunk inclusive of the aneurysm was performed with dual stent placement through each basilar and internal carotid artery across their respective junctions with the PTA. Six-month follow-up angiography showed complete occlusion of the PTA and the aneurysm. The patient's symptoms resolved. Our case demonstrated that the sacrifice of an adult type, Saltzman type 3 PTA inclusive of an associated fusiform aneurysm is feasible with dual stent-assisted coil embolization.

Key Words : Endovascular coil embolization; Fusiform aneurysm; Persistent trigeminal artery

Aneurysms of the persistent trigeminal artery (PTA) trunk proper are exceptionally rare. Dual stent placement supported endovascular coil embolization of a fusiform aneurysm with sacrifice of the parent PTA has not been previously described in the literature.

\footnotetext{
'Department of Neurosciences, St Joseph Medical Center, USA ${ }^{2}$ Department of Neurosciences, Advocate BroMenn Medical Center, USA

Received August 26, 2016; accepted after revision August 30, 2016. Correspondence to: Gordhan Ajeet, MD, Department of Neurosciences, St Joseph Medical Center, 2200 East Washington Street, Bloomington IL USA 61701

Tel. 1.309.662.3311 ext 4780 Fax. 1.309.662.3135

E-mail: gordhana@gmail.com

This is an Open Access article distributed under the terms of the Creative Commons Attribution Non-Commercial License (http://creativecommons.org/licenses/by-nc/3.0) which permits unrestricted non-commercial use, distribution, and reproduction in any medium, provided the original work is properly cited.
}

\section{CLINICAL PRESENTATION}

A 44-year-old female with chronic left sided retroorbital throbbing headache was identified to have a 9 $\mathrm{mm} \times 9 \mathrm{~mm} \times 6 \mathrm{~mm}$ fusiform aneurysm arising from medially coursing left adult type, Saltzman type 3 PTA trunk on diagnostic cerebral angiography (Fig. 1). No other vascular anomaly or intracranial aneurysm was identified. She had a 30 pack year history of smoking with no other significant comorbidities.

The endovascular procedure was performed under general anesthesia and full systemic heparinization. Pre-procedural antiplatelet medication with aspirin and clopidogrel was given for 5 days. For endovascular procedures, bi-femoral arterial access was utilized. A Benchmark guide catheter (Penumbra, Oakland, California, USA) was positioned within the left internal carotid artery (ICA) through the right common femoral artery access. A Neuron 070 guide catheter (Penumbra, 


\section{Gordhan Ajeet, et al.}

Oakland, California, USA) was positioned within the right vertebral artery through the left common femoral artery.

First, a Neuroform EZ Stent $(3.5 \mathrm{~mm} \times 20 \mathrm{~mm})$ (Stryker, Kalamazoo, MI, USA) was implanted through the basilar artery across the origin site of the left PTA confluence with the basilar artery. A second Neuroform EZ stent $(4.5 \mathrm{~mm} \times 20 \mathrm{~mm})$ was implanted through the left ICA across the origin site of left PTA confluence with the ICA (Fig. 2A). An Excelsior SL 10 (Stryker, Kalamazoo, MI, USA) micro-catheter was advanced through the basilar artery into the posterior segment of the PTA. Coil packing of the posterior efferent segment of the PTA was initially performed (Fig. 2B). The Excelsior SL 10 micro-catheter was then navigated into anterior afferent segment of the left PTA. Complete occlusion of the aneurysm lumen and the afferent segment with detachable coils was achieved, completing the sacrifice of the PTA (Fig. $2 \mathrm{C}$ and 2D). The post procedural course was uneventful. Six month angiographic follow up showed persistent occlusion of the aneurysm and the PTA (Fig. 3). The patient's symptoms resolved completely.

\section{DISCUSSION}

Fifteen percent of patients with PTA are likely to have aneurysms, but only 40 cases of such have been documented in the literature [1]. These aneurysms occur most commonly at the junctions with the ICA (type 1) or basilar artery (type 2) [1, 2, 3]. Aneurysms arising from the PTA trunk proper are exceptionally rare, with only 12 cases reported until 2004 [1]. These have been primarily saccular aneurysms with a high propensity to rupture. Such aneurysms have been surgically and endovascularly treated $[1,4,5]$. In the latter instance, partial sacrifice of the PTA was performed, due to concerns of compromising brainstem perforator vessels [5]. Endovascular treatment of a nonsaccular fusiform aneurysm of the PTA with sacrifice of the trunk inclusive of the aneurysm, by dual placement of stents through both ICA and basilar artery to protect parent vessels has not previously been reported.

Sacrifice of the PTA may cause ischemic injury to the brain stem due to occlusion of perforator vessels. An ipsilateral posterior communicating artery with fetal configuration is able to adequately collateralize the brainstem, if the patient had a PTA with an adult type anatomic configuration. This may not be the case in patients with the fetal type PTA, where the posterior circulation is mainly supplied from the PTA $[1,2]$. In cases of PTA with fetal type configuration, the risk of brainstem perforator infarcts can be potentially assessed by balloon occlusion testing [3].

PTA aneurysms are primarily asymptomatic $[1,2]$. Laterally coursing PTA may result more commonly in 6 th rather than 5 th cranial nerve palsies $[1,6]$. Symptoms may also arise from posterior circulation hypo-perfusion [2]. In treating our patient, posterior circulation coil embolization through the PTA was an important consideration [7]. Placement of coils only in the aneurysm would hypothetically result in thrombus formation at the afferent and efferent stumps of the trunk relative to the aneurysm, posing an embolic risk to the anterior and posterior circulations. Oizuka et al
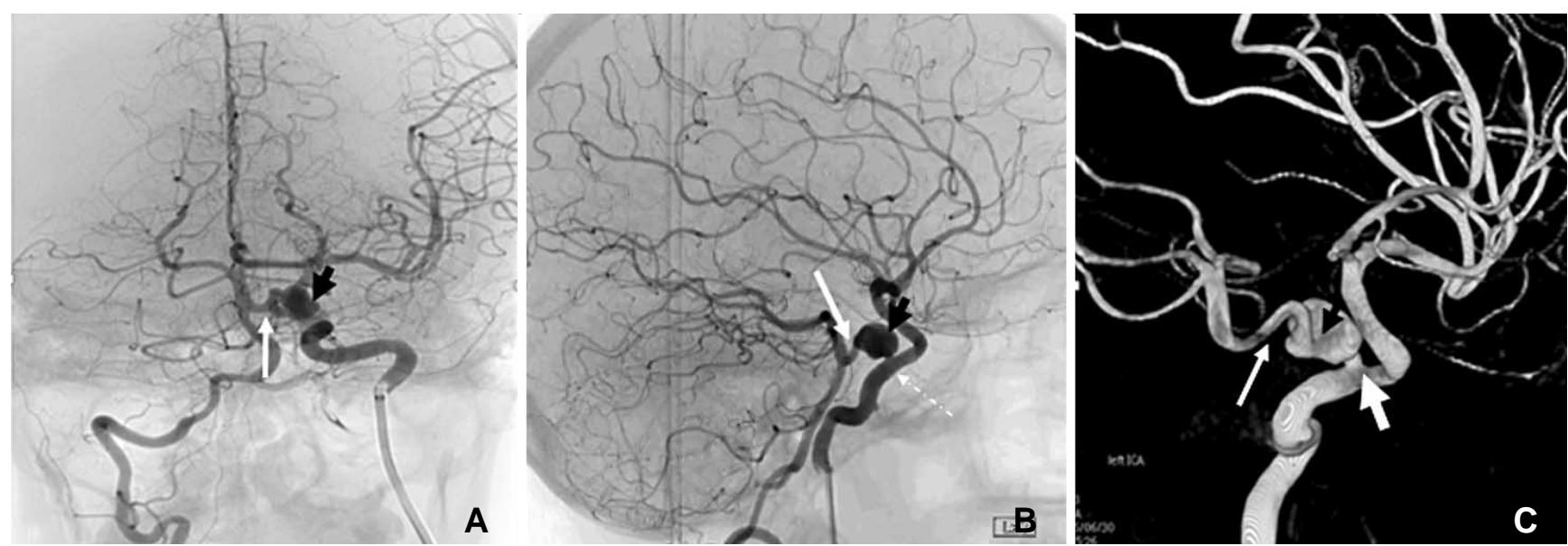

Fig. 1. Frontal (A) and lateral (B) projection un-subtracted Digital Subtraction Angiography (DSA) of simultaneous right vertebral and left ICA (dotted arrow) injections, demonstrate a medial coursing, adult type, Saltzman Type 3 Primitive Persistent Trigeminal Artery (PTA) (white arrow) with a $9 \mathrm{~mm}$ aneurysm centered within the trunk (black arrow). Surface shaded 3D rendition (C) of left ICA injection identifies the afferent (white thick) and efferent (thin white) segments of the trunk relative to the fusiform aneurysm (black arrow). 


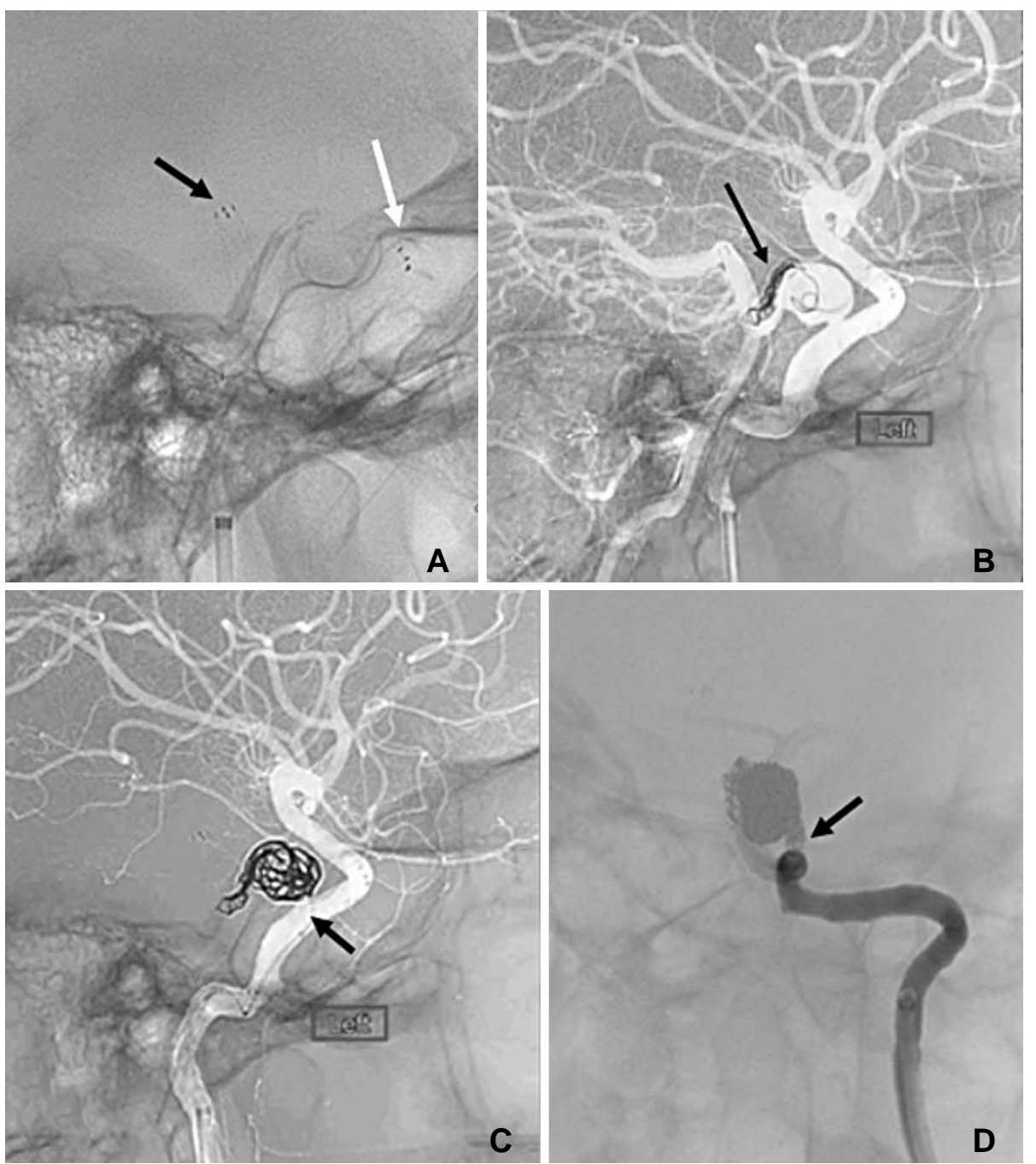

Fig. 2. Lateral projection fluoroscopic spot view (A) showing the Neuroform stents within the basilar artery (black arrow) and left ICA (white arrow). Lateral projection over-laid digital road map of simultaneous right vertebral and left ICA injections (B) demonstrating retrograde micro-catheter placement with coil embolization of the efferent PTA segment (black arrow). Lateral projection over-laid digital road map of left ICA injection $(\mathbf{C})$ showing antegrade micro-catheter placement with coil embolization of the aneurysm (black arrow). Frontal projection digital subtraction angiography of the left ICA (D) showing coil occlusion of the afferent aspect of the PTA (black arrow).
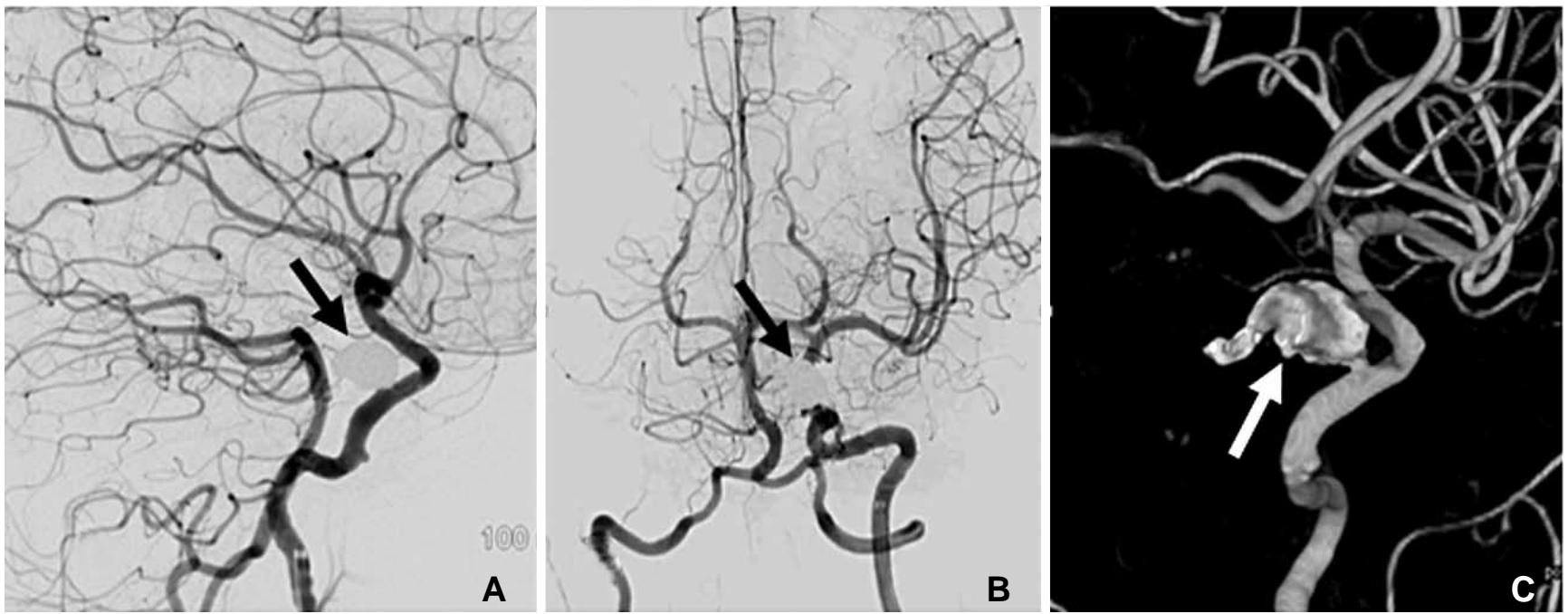

Fig. 3. Lateral (A) and frontal (B) projection Digital Subtraction Angiography (DSA) of simultaneous right vertebral and left ICA injections, demonstrate complete sacrifice of the PTA trunk and aneurysm (black arrow). Surface shaded 3D (C) rendition of left ICA injection shows occlusion of the aneurysm and trunk (white arrow). 


\section{Gordhan Ajeet, et al.}

reported on endovascular treatment of a PTA aneurysm resulting in coil prolapse into the ICA, which was subsequently managed by sacrifice of the ICA [3]. In our case, the Neuroform stents were implanted through the ICA and basilar artery at the junctions with the PTA, to avoid such a complication. In our case, retrograde coil embolization of the efferent limb through the basilar artery and the aneurysm as well as the afferent limb through the ICA resulted in complete durable occlusion and alleviation arising from the patient's symptoms. In conclusion, endovascular coil embolization of a fusiform aneurysm of an adult type, Saltzman type 3 PTA can be achieved safely with dual stent placement within each ICA and basilar artery and complete sacrifice of the PTA.

\section{References}

1. Ladner TR, Ehtesham M, Davis BJ, Khan IS, Ghiassi M, Ghiassi $\mathrm{M}$, et al. Resolution of trigeminal neuralgia by coil embolization of a persistent primitive trigeminal artery aneurysm. J Neurointerv
Surg 2014;6:e22

2. Aguiar GB, Conti ML, Veiga JC, Jory M, Souza RB. Basilar artery aneurysm at a persistent trigeminal artery junction. A case report and literature review. Interv Neuroradiol 2011;17:343-346

3. Onizuka M, Kazekawa K, Tsutsumi M, Kodama T, Aikawa H, Ikou M, et al. Hyperform remodeling balloon for the balloon occlusion test of persistent primitive trigeminal artery aneurysm-case report. Neurol Med Chir (Tokyo) 2006;46:541-543

4. Takase T, Tanabe H, Kondo A, Nonoguchi N, Tane K. Surgically treated aneurysm of the trunk of the persistent primitive trigeminal artery--case report. Neurol Med Chir (Tokyo) 2004;44:420-423

5. Hanabusa K, Murao K, Morikawa A, Taki W, Waga S. Endovascular treatment for a ruptured persistent trigeminal artery variant aneurysm on the distal portion--case report. Neurol Med Chir (Tokyo) 2000;40:637-640

6. Tsuboi K, Shibuya F, Yamada T, Nose T. Giant aneurysm at the junction of the left internal carotid and persistent primitive trigeminal arteries--case report. Neurol Med Chir (Tokyo) 1992;32:778781

7. Cloft HJ, Razack N, Kallmes DF. Prevalence of cerebral aneurysms in patients with persistent primitive trigeminal artery. $J$ Neurosurg 1999;90:865-867 